\title{
Statin use and peripheral blood progenitor cells mobilization in patients with multiple myeloma
}

\author{
A. Stravodimou • I. A. Voutsadakis
}

Received: 28 September 2012/ Accepted: 10 April 2013/Published online: 30 April 2013

(c) Federación de Sociedades Españolas de Oncología (FESEO) 2013

\begin{abstract}
Purpose Statins have beneficial effects in patients after myocardial infarction and at least part of the benefit results from mobilization of marrow endothelial progenitors to repopulate damaged myocardial tissues. This study examines if statins may have the same effect in mobilizing marrow progenitors to be harvested and subsequently used in high-dose chemotherapy with progenitor cell rescue in multiple myeloma.

Methods From 2006 to 2012, 86 patients with multiple myeloma were mobilized with the use of G-CSF and were retrospectively analyzed. Patients with other malignancies or mobilized with the use of chemotherapy or with plerixafor were excluded.

Results The median age of the patients was 60 years. 72 patients had received one line of chemotherapy and 14 patients two or more lines of chemotherapy. Twenty patients were taking statins at the time of the harvest while 66 patients were not. In the group of patients taking statins the success rate of first leukapheresis (obtaining the target number of $4 \times 10^{6} \mathrm{CD} 34+$ cells $/ \mathrm{kg}$ ) was $85 \%$ while in the group not taking statins this rate was $63.6 \%$. Despite the comparatively small number of patients this difference approached statistical significance $\left(\chi^{2}=0.07\right)$.

Conclusion This retrospective analysis of 86 patients shows for the first time a possible benefit of statins for
\end{abstract}

A. Stravodimou · I. A. Voutsadakis

Department of Medical Oncology, University Hospital of

Lausanne, Lausanne, Switzerland

\section{A. Voutsadakis $(\square)$}

Centre Pluridisciplinaire d'Oncologie, BH06, Centre Hospitalier

Universitaire Vaudois, Bugnon 46, 1011 Lausanne, Switzerland e-mail: ivoutsadakis@yahoo.com;

Ioannis.Voutsadakis@chuv.ch peripheral blood progenitor cells mobilization in patients with multiple myeloma. Larger studies would be required to clarify the issue. If their effectiveness is confirmed, statins could be a safe and cheaper addition to chemotherapy and plerixafor for peripheral hematopoietic stem cell mobilization.

Keywords Statins - Autologous transplantation . Mobilization · Multiple myeloma

\section{Introduction}

Statins are drugs that inhibit the enzyme 3-hydroxy-3methylglutaryl coenzyme A (HMG-CoA) reductase and are extensively used in the treatment of hypercholesterolemia to prevent cardiovascular events [1]. HMG-CoA reductase is the first enzyme of the steroids biosynthesis and prenylation pathways and catalyzes the production of mevalonate from $\mathrm{HMG}-\mathrm{CoA}$ [2]. In subsequent enzymatic reactions mevalonate is transformed to prenylation precursor geranyl pyrophosphate and to steroids precursor squalene. The beneficial effects of statins in patients with coronary artery disease and after myocardial infarction are believed to result, at least in part, from mobilization of marrow endothelial progenitors to repopulate damaged myocardial tissues [3]. Ischemic injury leads to the production of cytokines which triggers release of endothelial progenitors from the bone marrow [4]. In addition, progenitor cells expressing hematopoietic stem cell surface markers such as CD34, CD117 and CXCR4 are concomitantly released [5]. Statins promote the release of endothelial progenitor cells from bone marrow following myocardial infarction [6]. Such an effect in mobilization of progenitors, and specifically of hematopoietic progenitors, 
Table 1 Characteristics and outcomes of the whole group of patients studied and the two groups of patients based on whether they were taking statins at the time of leukapheresis

\begin{tabular}{|c|c|c|c|c|}
\hline & Total $(n=86)(\%)$ & Control $(n=66)(\%)$ & Statins $(n=20)(\%)$ & $\chi^{2}$ \\
\hline Age (year, median) & 60 & 60 & 61.5 & \\
\hline \multicolumn{5}{|l|}{ Sex } \\
\hline Male & $60(69.8)$ & $45(68.2)$ & $15(75)$ & \multirow[t]{2}{*}{$p=0.55$} \\
\hline Female & $26(30.2)$ & $21(31.8)$ & $5(25)$ & \\
\hline \multicolumn{5}{|l|}{ Salmon-Durie stage } \\
\hline I & $13(15.1)$ & $9(13.6)$ & $4(20)$ & \multirow[t]{3}{*}{$p=0.73$} \\
\hline II & $20(23.3)$ & $15(22.7)$ & $5(25\}$ & \\
\hline III & $53(61.6)$ & $42(63.6)$ & $11(55)$ & \\
\hline \multicolumn{5}{|l|}{ Previous lines of chemotherapy } \\
\hline 1 & $72(83.7]$ & $55(83.3)$ & $17(85)$ & \multirow[t]{2}{*}{$p=0.85$} \\
\hline$\geq 2$ & $14(16.3)$ & $11(16.7)$ & $3(15)$ & \\
\hline Success of 1st leukapheresis & $59(68.6)$ & $42(63.6)$ & $17(85)$ & $p=0.07$ \\
\hline $\mathrm{CV}$ treatments & $37(43\}$ & $24(36.3)$ & $13(65)$ & $p=0.02$ \\
\hline Median CD34+ count $\times 10^{6} / \mathrm{kg}$ & 5.2 & 5.0 & 6.3 & \\
\hline
\end{tabular}

The $\chi^{2}$ column gives the $p$ value of the comparison between the two groups

Control group patients not taking statins, $C V$ cardiovascular

would also be desirable in the setting of harvesting for subsequent autologous transplantation for the treatment of hematologic malignancies. The present study examines if statins may have an effect in the mobilization of marrow progenitors to the peripheral circulation in order to be harvested and subsequently used in high-dose chemotherapy with progenitor cell rescue in patients with multiple myeloma.

\section{Patients and methods}

Medical records of patients with multiple myeloma mobilized and harvested in the University Hospital of Lausanne in order to obtain hematopoietic stem and progenitor cells for subsequent autologous peripheral hematopoietic stem cell transplantation were reviewed. From 2006 to June 2012, 86 patients with multiple myeloma were mobilized with the use of G-CSF alone and were included in this retrospective analysis. Age and sex of the patients, type and stage of myeloma at diagnosis and previous therapies received were recorded. Administration of concomitant medications for cardiovascular diseases was noted. Information regarding the number of leukapheresis sessions and the number of mobilized CD34+ cells per $\mu$ l of peripheral blood the morning of leukapheresis in each patient and whether he or she was taking statins at the time of the procedures were also recorded. Patients with other malignancies or mobilized with the use of chemotherapy or with plerixafor were excluded from the analysis. Comparisons of the mean values of CD34+ mobilized cells in the two groups taking or not taking statins was done using the $t$ test. Associations of different prognostic parameters in the two groups were evaluated using the $\chi^{2}$ test. A logistic regression analysis was performed to test the influence of age, sex, stage, previous lines of anti-myeloma treatments, other concomitant cardiovascular medications and statins on the success of first leukapheresis.

\section{Results}

The median age of the patients was 60 years (range 37-72). There were 60 men and 26 women in the entire cohort of patients. $15.1 \%$ of the patients had Salmon-Durie stage I disease, $23.3 \%$ had Salmon-Durie stage II disease and $61.6 \%$ had stage III disease. Seventy-two patients had received one line of chemotherapy and 14 patients had two lines or more before mobilization. 48 patients had IgG immunoglobulin myeloma, 22 patients had IgA myeloma and 16 patients had light chains disease. Twenty patients were taking statins (atorvastatin, 9 patients; 4 at $40 \mathrm{mg} /$ day and 5 at $20 \mathrm{mg} /$ day, pravastatin, 5 patients; 4 at $20 \mathrm{mg} /$ day and 1 at $10 \mathrm{mg} /$ day and simvastatin, 6 patients; 4 at 40 $\mathrm{mg} /$ day and 2 at $20 \mathrm{mg} / \mathrm{day}$ ) at the time of the harvest while 66 patients were not taking HMG-CoA reductase inhibitors. The median age of the group taking statins was 61.5 years (range 45-72) while the median age of the control group (patients not taking statins) was 60 years (range 37-69). There was no statistically significant difference between the two groups in other parameters that could affect mobilization such as age, disease stage at 
diagnosis and previous therapies received (Table 1). Three patients in the statin group (15\%) and 11 in the control group $(16.7 \%)$ had received two or more lines of chemotherapy before mobilization $(p=0.85)$. No patient in either group has received more than four cycles of lenalidomide-containing regimens. Thirteen patients $(65 \%)$ were taking additional cardiovascular medications including anti-hypertensive, anti-arrhythmic, congestive heart failure medications, aspirin, coumadins and heparins in the statin group and 24 patients $(36.3 \%)$ were taking such medications in the control group $(p=0.02)$.

In the group of patients taking statins the target number of $4 \times 10^{6} \mathrm{CD} 34+$ cells $/ \mathrm{kg}$ could be obtained with a single apheresis session in 17 of 20 (85\%) patients (Table 1). From the three remaining patients, two required a second session to complete mobilization and one required three sessions. In the group not taking statins, in 42 of 66 patients $(63.6 \%)$ the target number of $4 \times 10^{6} \mathrm{CD} 34+$ cells $/ \mathrm{kg}$ was obtained with one session while the rest required more than one session to obtain the desired number. This difference shows a trend towards statistical significance $\left(\chi^{2}=3.25, p=0.07\right)$. In multivariate logistic regression analysis (Table 2), statin treatment and the number of previous chemotherapy lines (one versus two or more) were the only variables that approached statistical significance ( $p=0.06$ and 0.07 , respectively) while no trend for significance was observed for the other parameters including the age and the sex of the patients, the SalmonDurie stage of the disease and whether patients were receiving treatments for cardiovascular diseases. With additional apheresis sessions the target number of $4 \times 10^{6}$ CD34+ cells $/ \mathrm{kg}$ could be obtained in all patients taking statins and in 60 of 66 patients not taking statins. Thus, the overall failure rate was 0 and $9.1 \%$, respectively, $(p=0.16)$. Six patients in the non-statin group could not

Table 2 Logistic regression analysis with the success of first leukapheresis as the dependent variable and age sex stage of the disease (I and II versus III) previous lines of chemotherapy (one versus two or more), statin treatment and treatment with other cardiovascular drugs as the independent variables

\begin{tabular}{lllll}
\hline Variable & $\begin{array}{l}\text { Hazard } \\
\text { ratio }\end{array}$ & $\begin{array}{l}\text { 95 \% confidence } \\
\text { interval }\end{array}$ & $p$ value \\
\cline { 3 - 4 } & & $\begin{array}{l}\text { Lower } \\
\text { limit }\end{array}$ & $\begin{array}{l}\text { Upper } \\
\text { limit }\end{array}$ & \\
\hline Age & 0.9794 & 0.9191 & 1.0438 & 0.5223 \\
Sex & 1.2433 & 0.4308 & 3.5880 & 0.6871 \\
Stage & 1.3878 & 0.5139 & 3.7473 & 0.5179 \\
$\begin{array}{l}\text { Previous } \\
\text { chemotherapies }\end{array}$ & 0.2927 & 0.0766 & 1.1181 & 0.0724 \\
Statins & 3.6382 & 0.9067 & 14.5984 & 0.0685 \\
Other CV treatments & 0.7890 & 0.2746 & 2.2674 & 0.6599 \\
\hline
\end{tabular}

$C V$ cardiovascular be mobilized and had to receive alternative treatments without transplantation. In the 18 other patients, the required number was obtained with two sessions in 14 patients and with three sessions in four patients. The median number of cells harvested was $6.3 \times 10^{6} \mathrm{CD} 34+$ cells/kg in the group taking statins and $5.0 \times 10^{6} \mathrm{CD} 34+$ cells $/ \mathrm{kg}$ in the control group. The mean number of cells harvested $( \pm 95 \%$ confidence interval $)$ was $6.87 \pm$ $1.46 \times 10^{6} \mathrm{CD} 34+$ cells $/ \mathrm{kg}$ in the group taking statins and $6.06 \pm 1.05 \times 10^{6} \mathrm{CD} 34+$ cells $/ \mathrm{kg}$ in the control group (two-tailed $t$ test $p=0.36$ ). The mean absolute CD34+ cell count mobilized in the peripheral blood the morning of the first leukapheresis $( \pm 95 \%$ confidence interval) was $100.2 \pm 35.43 / \mu 1$ in the statin group and $84.1 \pm 16 / \mu 1$ in the control group (two-tailed $t$ test $p=0.35$ ). The duration of statin therapy in these patients was not systematically recorded and could not be ascertained from our retrospective data base.

\section{Discussion}

An effect of HMG-CoA reductase inhibitors in bone marrow progenitor cell mobilization has been suggested in preclinical models and in the clinic and possibly contributes to the beneficial effects of statins in myocardial infarction patients $[7,8]$. A study in pigs in which a coronary artery was rendered stenotic to produce hibernating myocardium showed that treatment with pravastatin for 5 weeks produced a dose-dependent increase of CD133+/c-kit+ cells in the circulation and in the diseased myocardium [7]. Both these markers are expressed not only by endothelial but also by hematopoietic progenitors. In a clinical study, treatment of patients undergoing a coronary stent placement with just 3 days of high-dose atorvastatin augmented the number of circulating endothelial progenitor cells by 3.5-fold [8].

Given these data, we examined in this retrospective study whether an effect in hematopoietic progenitor cell mobilization could be discernible in myeloma patients taking statins at the time of the procedure compared with patients not taking these drugs. Indeed, we observed that the success of the first leukapheresis increased from $63.6 \%$ in control patients not taking statins to $85 \%$ in patients taking statins. This effect approached but did not reach statistical significance $(p=0.07)$. In multivariate logistic regression analysis with the success of first leukapheresis as the dependent variable, treatment with statins and the number of previous lines of chemotherapy were the only parameters that approached statistical significance while the age and sex of the patients, the stage of myeloma and whether or not patients were receiving other drugs for cardiovascular diseases were not statistically significant. In 
addition, the median number of harvested cells increased in statin-treated patients and the overall failure rate decreased from almost $10 \%$ in controls to 0 . These effects in mobilization become more interesting if one takes into account that hypercholesterolemia and atherosclerotic vascular disease, conditions for which patients in the study were treated with statins, are risk factors for decreased progenitor mobilization [9]. In our series population, $65 \%$ of patients in the statin group and $36.3 \%$ of patients in the control group were taking other cardiovascular medications.

Hypercholesterolemia leads to accumulation of LDLs (low-density lipoproteins) in the subendothelial space of vessels and promotes atherosclerosis. Atherosclerosis is a risk factor for impaired stem cell mobilization and, as a result, its reversal may negate this impairment [10]. Atherosclerosis in the bone marrow microvasculature interferes with the local microenvironment of the perivascular niche where hematopoietic stem cells primed for mobilization reside [11]. Perivascular niche is part of the wellvascularized areas of bone marrow and may be more affected by vascular endothelial dysfunction characterizing hypercholesterolemia as opposed to the endosteal niche which is a less well-perfused area and where hematopoietic stem cells are maintained quiescent. Alternatively, both quiescent and primed progenitors residing in the two types of niches may be equally affected by vascular dysfunction, given their dynamic properties that allow them to pass from the one state to the other $[11,12]$. Monocyte and macrophage lineage cells constitute an important part of the atherosclerotic lesion becoming loaded with cholesterol and transformed to foamy cells. Monocytes are also part of the hematopoietic stem cell niches in the bone marrow and mediate the signal of mobilization by G-CSF [13]. As opposed to hematopoietic stem cells, monocytes express the receptor for G-CSF on their surface and, after ligation, signal to down-regulate adhesion molecules that retain adjacent stem cells in the niche [11]. Thus, dysfunction of monocytes in hypercholesterolemia could interfere with G-CSF-mediated mobilization.

Reversal of hypercholesterolemia may not be the only factor promoting stem cell mobilization by statins and other mechanisms may contribute. In fact hypercholesterolemia per se may promote hematopoietic cell mobilization and it is many times associated with neutrophilia in humans [14]. A study examining stem cell mobilization after highdose cyclophosphamide and G-CSF treatment found that hypercholesterolemic patients had better mobilization results than normocholesterolemic patients [15]. As statins interfere with cholesterol production leading to decreased plasma cholesterol, this cholesterol-lowering effect could be a factor that contributes to decreased mobilization. On the other hand, statins reversal of endothelial dysfunction would be expected to positively influence mobilization. Despite these contradictory influences of statins on cell mobilization, other effects stemming from decreased plasma cholesterol and impaired biosynthesis may be salutary for progenitor cells survival and readiness to be mobilized. Cholesterol biosynthesis is involved in the maturation process of myeloid progenitors and inhibition by statins may influence progenitor number and ability to interact with bone marrow microenvironment [16]. Lower plasma cholesterol decreases the availability of this lipid for incorporation in plasma membranes and could contribute to a re-organization of membrane domains with an effect on membrane content for growth factor receptors and adhesion molecules. Lipid rafts, membrane domains rich in cholesterol and sphingolipids are platforms for signaling emanating from the surface of cells by facilitating interactions of receptors and co-receptor molecules after interaction with ligands [17]. Interference with cholesterol biosynthesis and availability to be incorporated to membrane rafts may impede maturation of progenitors till robust signals such as pharmacologic doses of G-CSF used in the clinic for mobilization arrive.

The influence of statins in reversal of endothelial dysfunction accompanying atherosclerosis is mediated not only by their effect on cholesterol metabolism but also by cholesterol-independent actions. Nitric oxide (NO) is a bioactive molecule with strong vasodilator properties. Its production is increased by statins through activation of the endothelial NO synthase (eNOS) [18]. NO promotes bone marrow progenitors mobilization [19].

Statins have been reported to possess anti-myeloma effects in cell culture in vitro and in initial clinical trials and this could be an additional argument for using the drugs in myeloma patients in preparation for autologous transplantation [20]. In a small clinical study the addition of lovastatin to thalidomide and dexamethasone has improved progression-free survival and overall survival of relapsed or refractory myeloma patients compared with thalidomide and dexamethasone without lovastatin [20]. In another study, myeloma patients who underwent autologous transplantation and had used statins in the peritransplantation period had a trend towards a higher overall response rate (Complete Response, Very Good Partial Response and Partial Response) compared with patients who had not used statins during this period [21]. Although no study has reported the use of statins as mobilizing agents to date, it is interesting to note that in this last study [21] the number of stem cells mobilized in patients taking a statin in the peri-transplantation period was higher than in patients who were not (mean $8.3 \times 10^{6} \mathrm{CD} 34+$ cells $/ \mathrm{kg}$ versus $6.5 \times 10^{6} \mathrm{CD} 34+$ cells $\left./ \mathrm{kg}\right)$.

Previous therapy may interfere with the success of hematopoietic stem cell harvest in multiple myeloma 
patients. Specifically, protracted use of lenalidomide has been shown to decrease the success of leukapheresis while no clear effect of thalidomide or bortezomib has been documented [22, 23]. Thus, an expert panel guideline recommends hematopoietic progenitor harvest early in the course of such treatment preferably within the first four cycles [24]. The mechanism of this untoward lenalidomide effect does not appear to be due to direct stem cell toxicity, but may involve an increase in endogenous G-CSF concentrations that would lead to tachyphylaxis at the time of exogenous G-CSF administration for mobilization [25]. In our series no patient had received more than four cycles of lenalidomide-containing chemotherapy at the time of harvest.

The duration of statin treatment in our patient population could not be accurately ascertained from our retrospectively examined records. Nevertheless, this duration may not be critical for the possible mobilization effect given that in coronary artery disease patients undergoing coronary stent placement, progenitor cell mobilization was observed with just 3 days of treatment [8]. A blunting of the mobilization effect is noted with longer treatments $[8$, 26]. These data may also imply that a brief treatment with statins for a few days prior to harvest in myeloma patients could be sufficient to derive the mobilization benefit.

In conclusion, this retrospective analysis of 86 patients discloses a numerically important difference in the success of peripheral blood progenitors harvest in patients taking statins with a trend towards statistical significance. This is clearly a hypothesis-generating investigation and larger studies would be required to clarify the issue of a possible beneficial effect of statins in hematopoietic progenitor cell mobilization. These studies should optimally be prospective and randomized and include sufficient patients to show an effect. Based on the magnitude of the benefit seen in our study, 180-200 patients could be sufficient. Only patients not taking statins at the time of randomization or recently before would be eligible. Treatment could be as brief as several days and an escalation period up to the maximal recommended dose for hypercholesterolemia (for which extensive data on safety exist) could precede the full dose period. A short period of overall treatment would have the additional advantage to be practical as it would not significantly interfere with the timetable of the standard autologous transplantation treatment for these patients. In addition, it would avoid any undesirable effect associated with long-term statin treatment such as myopathy and increased diabetes risk [27]. A translational component to elucidate the mechanisms of the possible mobilization effect would be highly desirable. This should include an analysis of adhesion molecules and membrane domains on mobilized progenitors and the effects of statins on them. If their effectiveness is confirmed, statins could be a safe and cheaper addition to chemotherapy and plerixafor for peripheral hematopoietic stem cell mobilization in multiple myeloma and possibly other hematologic malignancies.

\section{Conflict of interest None.}

\section{References}

1. Minder CM, Blaha MJ, Horne A, Michos ED, Kaul S, Blumenthal RS. Evidence-based use of statins for primary prevention of cardiovascular disease. Am J Med. 2012;125:4440-6.

2. Thurnher M, Nussbaumer O, Gruenbacher G. Novel aspects of mevalonate pathway inhibitors as antitumor agents. Clin Cancer Res. 2012;18:1-8.

3. Urbich C, Dimmeler S. Risk factors for coronary artery disease, circulating endothelial progenitor cells, and the role of HMG-CoA reductase inhibitors. Kidney Int. 2005;67:1672-6.

4. Wojakowski W, Landmesser U, Bachowski R, Jadczyk T, Tendera M. Mobilization of stem and progenitor cells in cardiovascular diseases. Leukemia 2012;26:23-33.

5. Massa M, Rosti V, Ferrario M, Campanelli R, Ramajoli I, Rosso R, et al. Increased circulating hematopoietic and endothelial progenitor cells in the early phase of acute myocardial infarction. Blood. 2005;105:199-206.

6. Dimmeler S, Aicher A, Vasa M, Mildner-Rihm C, Adler K, Tiemann M, et al. HMG-CoA reductase inhibitors (statins) increase endothelial progenitor cells via the PI3-kinase/Akt pathway. J Clin Invest. 2001;108:391-7.

7. Suzuki G, Iyer V, Cimato T, Canty JM Jr. Pravastatin improves function in hibernating myocardium by mobilizing $\mathrm{CD}_{13} 3^{+}$and $\mathrm{cKit}^{+}$bone marrow progenitor cells and promoting myocytes to reenter the growth phase of the cardiac cell cycle. Circ Res. 2009;104:255-64.

8. Hibbert B, Ma X, Pourdjabbar A, Simard T, Rayner K, Sun J, et al. Preprocedural atorvastatin mobilizes endothelial progenitor cells: clues to the salutary effects of statins on healing of stented human arteries. PLoS ONE. 2011;6:e16413.

9. Werner N, Nickenig G. Influence of cardiovascular risk factors on endothelial progenitor cells: limitations for therapy? Arterioscl Thromb Vasc Biol. 2006; 26:257-66.

10. Vasa M, Fichtlscherer S, Aicher A, Adler K, Urbich C, Martin H, et al. Number and migratory activity of circulating endothelial progenitor cells inversely correlate with risk factors for coronary artery disease. Circ Res. 2001;89:E1-7.

11. Ehninger A, Trumpp A. The bone marrow stem cell niche grows up: mesenchymal stem cells and macrophages move in. J Exp Med. 2011;208:421-8.

12. Trumpp A, Essers M, Wilson A. Awakening dormant haematopoietic stem cells. Nat Rev Immunol. 2010;10:201-9.

13. Christopher MJ, Rao M, Liu F, Woloszynek JR, Link DC. Expression of the G-CSF receptor in monocytic cells is sufficient to mediate hematopoietic progenitor mobilization by G-CSF in mice. J Exp Med. 2011;208:251-60.

14. Suratt BT, Fessler MB. Greasing the way: the ABCs of HSPC efflux from the marrow. Cell Stem Cell. 2012;11:143-4.

15. Crysandt M, Hilgers RD, von Hobe S, Eisert A, Jost E, Panse J, et al. Hypercholesterolemia and its association with enhanced stem cell mobilization and harvest after high-dose cyclophosphamide + G-CSF. Bone Marrow Transplant. 2011;46:1426-9.

16. Subramanian G, Chaudhury P, Malu K, Fowler S, Manmode R, Gotur D, et al. Lamin $\mathrm{B}$ receptor regulates the growth and maturation of myeloid progenitors via its sterol reductase domain: implications for cholesterol biosynthesis in regulating myelopoiesis. J Immunol. 2012;188:85-102.

17. Lemaire-Ewing S, Lagrost L, Néel D. Lipid rafts: a signaling platform linking lipoprotein metabolism to atherogenesis. Atherosclerosis. 2012;221:303-10.

18. Preston Mason R, Walter MF, Jacob RF. Effects of HMG-CoA reductase inhibitors on endothelial function. Role of microdomains and oxidative stress. Circulation. 2004;109:34-41.

19. Aicher A, Heeschen C, Mildner-Rihm C, Urbich C, Ihling C, Technau-Ihling K, et al. Essential role of endothelial nitric oxide synthase for mobilization of stem and progenitor cells. Nat Med. 2003;9:1370-6.

20. Hus M, Grzasko N, Szostek M, Pluta A, Helbig G, Woszczyk D, et al. Thalidomide, dexamethasone and lovastatin with autologous stem cell transplantation as a salvage immunomodulatory therapy in patients with relapsed and refractory multiple myeloma. Ann Hematol. 2011;90:1161-6.

21. Hamadani M, Hade E, Benson DM Jr, Hofmeister CC. The effect of statin use at the time of autologous transplant on response and survival in multiple myeloma. Biol Blood Marrow Transplant. 2008;14:351-2.

22. Popat U, Saliba R, Thandi R, Hosing C, Qazilbash M, Anderlini P, et al. Impairment of filgrastim-induced stem cell mobilization after prior lenalidomide 
in patients with multiple myeloma. Biol Blood Marrow Transplant. 2009;15: 718-23.

23. Rajkumar SV, Blood E, Vesole D, Fonseca R, Greipp PR, Eastern Cooperative Oncology Group. Phase III clinical trial of thalidomide plus dexamethasone compared with dexamethasone alone in newly diagnosed multiple myeloma: a clinical trial coordinated by the Eastern Cooperative Oncology Group. J Clin Oncol. 2006;24:431-6.

24. Kumar S, Giralt S, Stadtmauer EA, Harousseau JL, Palumbo A, Bensinger W, et al. Mobilization in myeloma revisited: IMWG consensus perspectives on stem cell collection following initial therapy with thalidomide-, lenalidomide-, or bortezomib-containing regimens. Blood. 2009;114:1729-35.
25. Koh KR, Janz M, Mapara MY, Lemke B, Stirling D, Dörken B, et al. Immunomodulatory derivative of thalidomide (IMiD CC-4047) induces a shift in lineage commitment by suppressing erythropoiesis and promoting myelopoiesis. Blood. 2005;105:3833-40.

26. Hristov M, Fach C, Becker C, Heussen N, Liehn EA, Blindt EA, et al. Reduced numbers of circulating endothelial progenitor cells in patients with coronary artery disease associated with long-term statin treatment. Atherosclerosis. 2007;192:413-20.

27. Dirks AJ, Jones KM. Statin-induced apoptosis and skeletal myopathy. Am J Physiol Cell Physiol. 2006;291:C1208-12. 